\title{
LA RESPUESTA CORRECTA ÚNICA Y LA JUSTIFICACIÓN DE LA DECISIÓN JURÍDICA
}

\section{Introducción}

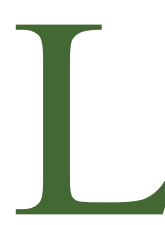

a expresión «respuesta correcta única» puede ser considerada en el razonamiento jurídico desde dos puntos de vista: un punto de vista interno, es decir, como respuesta lógicamente adecuada a un conjunto de premisas y reglas de derivación, y desde un punto de vista externo, es decir, como la decisión que armoniza satisfactoriamente puntos de vista antagónicos. Cada uno de estos puntos de vista requiere de una estructura de justificación cuyos elementos difieren sustancialmente entre sí.

A partir de la distinción entre lo que puede considerarse una respuesta jurídica lógicamente correcta, y lo que es una decisión jurídica justificable, en el presente trabajo me propongo en primer lugar establecer el significado de la expresión «respuesta correcta única» en ambos contextos; por otra parte también pretendo analizar los elementos que caracterizan una justificación suficiente en el campo de las decisiones jurídicas.

Voy a considerar el proceso que lleva al juez a producir una decisión dentro del marco general de la teoría de la decisión racional, con el fin de caracterizar los rasgos especiales de la elaboración de esa decisión, y también los relativos a la estructura de justificación de la misma. En este sentido es importante tener en cuenta que aunque la decisión jurídica incluya de manera necesaria el proceso de inferencia jurídica, como búsqueda de la respuesta correcta dentro de un sistema de premisas, aquélla no es co-extensiva con ésta, y demanda de una justificación vinculada con los elementos propios de la toma de decisión bajo incertidumbre.

En buena parte del desarrollo de mi punto de vista, mantendré diversos diálogos con el trabajo de Manuel Atienza «Para una Teoría de la Argumentación Jurídica», en el que el autor considera el problema de la relación entre los métodos de repre- 
sentación de la argumentación y la argumentación misma; y más extensamente con el trabajo de Aulis Aarnio «One Right Answer and the Regulative Principle of Legal Reasoning». En este trabajo Aarnio procura demostrar por qué es importante la justificación del razonamiento jurídico, y cómo puede alcanzarse la misma. Su punto de partida se localiza en la llamada «actitud crítica», que niega la posibilidad (ontológica y epistemológica) de una única respuesta correcta en el razonamiento jurídico; a pesar de lo cual, según Aarnio, puede llegarse a una justificación del mismo.

Voy a sostener que la elaboración y justificación de una decisión jurídica posee una estructura y un procedimiento que le es propio, y que dicha estructura se diferencia de la de otras instancias del razonamiento jurídico. Así, mientras un juicio acerca de su coherencia es suficiente para la justificación de una inferencia jurídica, en el caso de la decisión jurídica la evaluación de la misma además del señalado juicio de coherencia, se vincula a un complejo proceso de justificación que es inseparable del momento de la construcción de la decisión.

\section{La Inferencia Jurídica: La respuesta correcta en el marco de un sistema}

El marco básico para el razonamiento jurídico es el sistema de normas, cuyos rasgos particulares van a influir en los métodos a ser empleados en la elaboración y justificación de la inferencia jurídica. Tales peculiaridades se refieren especialmente a la coherencia, extensión, y tipo de orden que caracteriza a dicho sistema. Las características propias del sistema de normas hacen que en los procesos de derivación sólo pueda emplearse un álgebra blanda que no ofrece soluciones puntuales, sino solamente las soluciones lógicamente posibles dentro del sistema [Barragán/89-a]. Las llamadas soluciones lógicamente posibles son aquellas que guardan coherencia con las premisas utilizadas y con las reglas de derivación aceptadas dentro del sistema. A fin de determinar el alcance de la justificación que puede lograrse en el ámbito de la inferencia jurídica, examinaremos el concepto de solución en la Teoría General del Comportamiento Racional.

Dentro del marco de la Teoría General del Comportamiento Racional el concepto de solución es extremadamente importante tanto desde el punto de vista descriptivo como normativo. Considerado en su aspecto normativo, el concepto presenta un procedimiento o grupo de procedimientos (llamados solución) que 
ofrecen buenas razones para actuar conforme a ellos; y en su aspecto descriptivo, la teoría provee de una caracterización acerca de las condiciones bajo las cuales la solución es posible.

Formalmente la solución consiste en un conjunto de restricciones, que definen el espacio de soluciones posibles, y una regla de decisión que permite elegir la llamada solución única. Tanto las restricciones como la regla de decisión pueden ser cambiadas, con lo que un mismo problema puede ser resuelto en un sentido u otro, según sea el marco considerado. Como es natural, la coherencia de una solución se encuentra siempre referida sólo a su marco de referencia; y según puede inferirse de la existencia de diversas soluciones únicas en problemas de negociación [Nash/50, Kalai-Smorodinski/75], en el ámbito de la Teoría de La Acción Racional el concepto de solución única tiene sentido estrictamente dentro de un marco de restricciones (incluidas las premisas seleccionadas) previamente aceptado, por lo que no puede ser empleado como justificación externa en una decisión. Esto último requeriría de una valoración exhaustiva de las restricciones establecidas, de las reglas de derivación empleadas, como asimismo de las consecuencias prácticas que la aceptación de las mismas pueden llegar a producir. Esta última evaluación traerá como consecuencia preferir una solución coherente a otra solución coherente, en razón de considerarla más aconsejable para el logro de un determinado fin.

El concepto de solución única, en consecuencia, puede ser de gran utilidad en el campo de la inferencia jurídica, ya que ayuda en principio a eliminar las soluciones que son incoherentes con las premisas y reglas de derivación aceptadas; pero supone un acuerdo previo acerca de éstas que no siempre corresponde con la realidad. Sin embargo, cuando se la alcanza, la solución única funciona como una información especialmente relevante en la toma de decisiones jurídicas.

\section{La Decisión Jurídica: Selección de la mejor alternativa}

Tal como lo hace Aarnio en el trabajo citado, vamos a considerar al juez como «un decisor racional que bajo condiciones de incertidumbre procura maximizar la certidumbre legal». El concepto de decisor racional es clave en la Teoría del Comportamiento Racional, y se aplica al decisor que es capaz de ordenar de manera clara y consistente las prioridades o preferencias involucradas en la decisión; de modo que al momento de seleccionar entre soluciones alternativas, lo hace basándose en una cuidado- 
sa evaluación de las ventajas y desventajas que tal selección involucra.

El valor cuya maximización se procura mediante la decisión es siempre el punto de referencia para evaluar tales ventajas y desventajas. Así, en la toma de decisiones individuales, en la medida que se persiguen fines también individuales, se procurarán maximizar las utilidades individuales; mientras que en las decisiones en el entorno social el decisor debe maximizar una determinada utilidad social, para lo cual tiene que armonizar puntos de vista antagónicos, incluido el suyo propio. Este tipo de decisión implica dificultades mayores que el de las decisiones individuales, ya que a los problemas generales de ordenamiento coherente se unen los derivados de la interacción de posiciones individuales. El primer tipo de decisiones se escenifica en el terreno de la Teoría de la Utilidad y de la Decisión, mientras que las segundas se ubican en el campo de la Teoría de los Juegos y de la Ética [Harsanyl/ 88].

En el caso particular del decisor jurídico, el valor social a maximizar sería el de la certidumbre legal; meta que como es natural, se logra reduciendo la incertidumbre. Asimismo, resulta claro que esta decisión se escenifica en el terreno de la interacción social, razón por la cual deberá confrontar todos los problemas que caracterizan ese tipo especial de decisión.

Dentro de ese particular campo corresponde preguntarse qué tipo de justificación puede considerarse suficiente para una toma de decisión bajo incertidumbre en el entorno social, cuando la misma procura maximizar la certeza legal. En el caso de la justificación interna de la inferencia jurídica el de la coherencia representa un ámbito suficiente para evaluar el razonamiento; pero en el caso de la decisión parece claro que no es suficiente. En efecto, toda decisión tiene por objeto transformar un mundo previo en otro posterior, modificado precisamente por efecto de esa decisión. En este proceso, la coherencia lograda en la inferencia jurídica juega un papel necesario pero no suficiente para la justificación de la decisión, ya que en ésta la base argumental es mucho más amplia, incluyendo datos de origen empírico, valorativo, político, ético, etc. [Barragán/ 89-b].

El hecho de que la decisión jurídica sea interactiva, transformadora del mundo, y de base poliargumental, hace que la justificación de la misma esté internamente ligada con el proceso de construcción de la decisión; esto equivale a decir que no es posible producir una justificación aceptable de una decisión que ya ha sido construida con anterioridad, si en ella no se han respetado los procedimientos que la legitiman. De manera que el análi- 
sis del proceso de construcción de la decisión es imprescindible si se desea determinar si la misma está justificada o no respecto de la meta general de maximización de la certidumbre jurídica. Por esta razón encuentro inadecuada la expresión que Aarnio usa en su trabajo, cuando al referirse a la mejor justificación posible la describe como «un intento de convencer a otra persona del valor de las razones.» El hecho de que se hable de «convencer» disocia el momento de la construcción del momento de la justificación de una manera inaceptable en el marco de una decisión interactiva bajo incertidumbre. Esta disociación tiene importantes repercusiones en lo que luego pueda considerarse la mejor justificación posible, y se corre el riesgo de reducir la justificación de la decisión jurídica a un mero intercambio de verbalizaciones posterior a la decisión misma.

El hecho de que la decisión judicial sea una decisión interactiva bajo incertidumbre genera algunas consecuencias importantes. En primer lugar supone que la misma debe elaborarse a través de un proceso de interacción que naturalmente incluye la idea general de diálogo como intercambio activo de opiniones dentro de un marco semántico; pero la idea de interacción rebasa a la de diálogo, ya que incluye otro tipo de intercambios. Esto cobra un sentido más claro si se considera que toda decisión bajo incertidumbre se inicia con una creencia inicial (probabilidad a priori) apoyada en la información que el decisor tiene acerca de un problema [De Finetti/89 y Savage/70 y Press/89]. En el caso de la decisión jurídica esta información incluye el conjunto de las posibles respuestas coherentes con el sistema de normas, pero además incluye también información acerca de circunstancias empíricas, acerca de los valores, creencias e intereses implicados en la decisión (incluidos los del propio decisor). En el proceso de interacción, tal información será apropiadamente corregida y objetivada, para dar lugar a nuevas creencias (probabilidad a posteriori), en sucesivos momentos. El intercambio entre el decisor y el destinatario de la decisión no es sólo un intercambio que tiende a «disminuir la diferencia entre sus opiniones» (Aarnio), sino que su finalidad es la de reducir sustancialmente la incertidumbre jurídica, meta que le hemos fijado inicialmente al decisor, y que va mucho más allá de lograr una argumentación aceptable en un caso determinado o en la interpretación de una normativa. En cierto tipo de decisiones jurídicas la fuerza de la interacción puede aparecer un tanto diluida; pero en otras, como por ejemplo las referidas al establecimiento de una ley determinada, o aquellas en que los insumos materiales son complejos y de origen muy diverso, la vitalidad del intercambio interac- 
tivo es lo único que puede ayudar a la buena elaboración y justificación de la decisión jurídica.

Naturalmente que todo el proceso interactivo de construcción de una decisión jurídica se hará más transparente, y en consecuencia contribuirá mejor a modificar la probabilidad a priori, si es pública y ampliamente argumentado. En este sentido la publicidad y la exposición abierta de razones constituyen una herramienta poderosa para corregir y ponderar con mayor acierto la información incorporable en la decisión. Pero esa publicidad no es sólo un factor de control de las razones expuestas acerca de una decisión ya elaborada, sino que es una fuente compleja, e infinitamente sustanciosa de modificación de la probabilidad que el decisor asigna a una determinada alternativa al momento de elaborar la decisión. Por esta razón no puedo coincidir con Aarnio cuando para señalar la importancia de las «razones abiertamente escritas» (públicas) lo hace sobre la base de que «de otra manera no podemos controlar el procedimiento real de razonamiento y sus resultados». De lo que se trata es de dar cabida en la elaboración de la decisión al mayor número posible de correctores de la probabilidad a priori. Pero este rol de la publicidad no puede ser percibido si se escinde artificialmente el momento de construcción de la toma de la decisión del de su justificación. En el marco de la toma de decisión racional no hay un momento de construcción y otro diferente de justificación, sino que esta última obtiene su aceptabilidad a partir del modo en que aquélla se produce. Esto significa que la justificabilidad de una decisión jurídica no se deriva de una buena exposición de razones (incluso si ella no apela a ninguna forma de coerción o manipulación), sino de haber reducido efectivamente la incertidumbre jurídica. En consecuencia la mejor justificación no es aquella de cuyo proceso se han eliminado los factores que puedan entorpecerla (Aarnio), sino la que surge de haber eliminado del proceso de construcción de la decisión los factores que pueden perturbar la reducción de la incertidumbre.

\section{Sobre el Hércules dworkiniano y otros Hércules...}

El Super-Juez de Dworkin mencionado por Aarnio, Hércules J., parece haber resuelto todos los problemas de incertidumbre, ya que no sólo posee información ilimitada acerca del asunto a decidir, sino que también dispone de todo el tiempo y la capacidad necesarios para tomar la decisión. Bajo estas condiciones podría esperarse que Hércules J. logre una respuesta jurídica úni- 
ca, y que además pueda exhibir muy buenas y coherentes razones para justificar tal respuesta. Si nos estuviésemos refiriendo sólo a una inferencia jurídica, esto sería aceptable, en la medida en que Hércules J. con un algoritmo (finito) suficientemente alimentado y operado durante el tiempo necesario, se empeñara en esa búsqueda; pero como el problema de que se trata es el de producir una decisión jurídica, Hércules J. no puede comportarse como un operador de algoritmos sino como un decisor racional cuya meta es la maximización de la certeza legal. En el propio momento en que Hércules J. debe enfrentarse a una toma de decisión interactiva bajo incertidumbre, su panorama se complica, y los niveles de probabilidad de lograr una respuesta correcta única caen abruptamente como resultado de la interacción, que llega acompañada de intereses antagónicos, de valoraciones diversamente fundadas, y de un manejo desigual de las bases materiales de la información.

Respecto de Hércules J. coincido con la afirmación de Aarnio (aunque no tanto con sus argumentos) acerca de que «es imposible aún para Hércules J. detectar una única respuesta correcta, y en consecuencia es imposible argumentar acerca de ella»; y es imposible porque las poderosas herramientas de que Hércules J. ha sido idealmente dotado (disponibilidad de información ilimitada, tiempo ilimitado, capacidad, etc.) están especialmente diseñadas para facilitar la inferencia, pero su utilidad es limitada en el momento de construir decisiones. Tales herramientas no ayudan a Hércules J. a discutir acerca del valor de sus propios puntos de partida (premisas), como tampoco pueden ayudarlo a articular los intereses discrepantes de los afectados por la decisión, ni a seleccionar en el espacio de respuestas coherentes con el sistema de normas aquella más valiosa desde el punto de vista de la ética colectiva. Tales herramientas no pueden entonces ayudar a Hércules J. en el proceso de alcanzar su meta como decisor, es decir, maximizar la certidumbre jurídica; y si tal como lo creemos el proceso de construcción de la decisión es inseparable del de justificación de la misma, tampoco en este último ámbito Hércules J. podrá alcanzar el éxito.

Pero cabría preguntarse si esa situación ideal de que se dota a Hércules J. cumple alguna función de importancia en la estructura de justificación de la decisión jurídica, además de la ya señalada de facilitar el momento de la inferencia jurídica. Aarnio se inclina por considerar que la situación ideal «Hércules J.» u otra situación ideal análoga cumplen la función crucial de «ofrecer el criterio para la crítica. Sin tal medida es imposible, por ejemplo, evaluar decisiones». Y esta afirmación sirve a Aarnio 
como base para introducir la situación ideal Comunidad jurídica II como criterio de evaluación de la decisión jurídica.

Habría que señalar que para Aarnio la Comunidad jurídica II es un constructo ideal que consiste en el conjunto de todos aquellos que se ciñen a las reglas de la Racionalidad Discursiva, distinguiéndola de la Comunidad jurídica I que es la comunidad concreta que existe en la práctica jurídica. La Comunidad Jurídica II como ya dijimos cumple para Aarnio un papel decisivo en la evaluación de la decisión jurídica, ya que la meta del decisor jurídico sería la de «tratar de alcanzar una solución y una justificación tales que la mayoría de las personas racionalmente pensantes pueda aceptarla como una solución propia».

Se pueden hacer algunos comentarios sobre este principio de Aarnio. Si la Comunidad jurídica II es una construcción ideal tal como él lo señala, entonces hay que aceptar que tal Comunidad es un cuerpo de conceptos que pueden ayudarnos a buscar y lograr una solución, pero utilizando esta palabra sólo en el sentido de las soluciones únicas de Nash o de Kalai-Smorodinski, es decir, como solución dentro de ese cuerpo de conceptos. Desde luego que en ningún caso puede ayudarnos a decidir acerca de cuál es la mejor solución, con lo que no serviría como criterio externo de justificación de una decisión. El propio Aarnio cuando analiza la función de la Racionalidad-D en el resultado final de una decisión, sostiene que éste «no depende solamente de los standards de racionalidad... sino también de los insumos (materiales de la decisión)... Aún más, (el resultado final) está conectado también con cosas distintas de la racionalidad, como por ejemplo los deseos e intereses del decisor». De esto se puede inferir que tanto las bases materiales de la decisión como los deseos e intereses del decisor son considerados independientes de la Racionalidad-D, y en consecuencia de la Comunidad jurídica II, con lo que ésta quedaría descalificada como criterio de justificación de la decisión jurídica.

Por otra parte, es poco claro qué puede significar en una construcción ideal como la Comunidad jurídica II la regla de la mayoría utilizada por Aarnio. Aun si aceptamos la difícilmente aceptable idea de que tiene sentido hablar de mayoría refiriéndose a un cuerpo de conceptos, quedaría otro problema por superar: los elementos ideales del conjunto, a pesar de que adhieren a las obligaciones derivadas de la Racionalidad-D, son sin embargo definidos en otros aspectos como no homogéneos, y pueden representar códigos morales muy diferentes. Esta circunstancia tiene una relevancia muy especial, ya que la regla de la mayoría no es buen criterio para utilizar en poblaciones heterogéneas; y 
si la heterogeneidad se observa en una variable tan relevante en la decisión como lo es el código moral, la regla sería inaplicable. Además, si la Comunidad jurídica II es definida como homogénea sólo en lo relativo a la racionalidad, insistir en utilizarla como criterio de justificación de la decisión, equivaldría a reducir esta última al solo ámbito de la racionalidad, alternativa que el propio Aarnio rechazaría en el terreno de la decisión jurídica.

El problema que presenta Hércules J. y otros modelos ideales como la Comunidad jurídica II, es que al tratar con una situación ficticia, no poseen (por definición) la capacidad informativa necesaria para reducir la incertidumbre de una manera real; de modo que cuando se los emplea en la evaluación de la decisión jurídica, esta evaluación se convierte en una mera comparación de la decisión con un modelo conceptual normativo, pero no en un proceso de reducción de la incertidumbre jurídica. Pareciera que sólo la información real aportada en los procesos de interacción es capaz de corregir la probabilidad a priori del decisor.

\section{Los mecanismos de la justificación en una decisión jurídica}

Hasta el momento hemos sostenido que ningún constructo ideal puede ayudar a modificar la probabilidad a priori del decisor jurídico, ya que tal modificación sólo puede producirse mediante la incorporación de información relevante al problema de decisión de que se trate. Entendido este proceso de incorporación como comprensivo de los dos aspectos que Atienza señala: agregación y/ o eliminación respecto de la información preexistente.

Ahora bien, esa incorporación de la información tiene por finalidad corregir la probabilidad a priori del decisor, probabilidad que ha sido definida en base a datos de origen empírico, teórico, normativo y valorativo. Tal probabilidad a priori llevaría al decisor de buena fe, a preferir una alternativa a otra; esto significa, en los diferentes ámbitos en que se desenvuelve el razonamiento jurídico, inclinarse por una determinada interpretación, o dictar una sentencia específica, o proteger en una ley un interés y no otro. Sin embargo, la probabilidad a priori no es certeza, y aun en el caso que lo fuera, el solo hecho de plantearse un proceso de construcción de una decisión, indica la disposición del decisor a corregirla a la vista de cada nueva información relevante, que puede provenir de los orígenes ya señalados.

En este punto coincido en lo sustancial con Atienza cuando sostiene que bajo ciertos supuestos... «argumentar sólo puede 
significar añadir nuevas informaciones o bien eliminar informaciones preexistentes»... Debo señalar que en el ámbito de la decisión jurídica la definición de Atienza es aplicable a todo asunto no trivial, porque de lo contrario el mismo no estaría sujeto a decisión.

Este papel corrector de la probabilidad a priori que cumple la información, determina que el tiempo en el que la misma tiene que ser incorporada no puede ser arbitrario, sino que debe darse en el interior del proceso de construcción de la decisión. Esto se debe a que en el momento en que la decisión queda tomada, no sólo se agota el proceso de su construcción sino también el proceso de su justificación. La argumentación se presenta entonces como el intercambio activo entre el decisor y otra parte interesada o afectada por la decisión, que permite construir la probabilidad a posteriori que dará lugar a la elaboración de la decisión.

En cuanto a las bases argumentales más utilizadas en la elaboración de una decisión jurídica, se encuentran las empíricas, las derivadas del sistema de normas, las derivadas de principios valorativos, y las derivadas de constructos conceptuales. Por otra parte, la decisión está también influenciada por todas las formas de condicionamiento del decisor que lo llevan a ubicarse en una perspectiva inicial determinada, cosa que también sucede con los destinatarios de la decisión.

El mejor método para la corrección de las bases argumentales y para la objetivación de los condicionamientos que operan en el proceso de decisión, pasa por el carácter interactivo de la toma de decisión en el entorno social. Sin la interacción (estimulada por la publicidad de tal proceso) en la construcción de la decisión, muy probablemente los elementos correctores de la probabilidad a priori tenderían a adquirir importantes e inevitables sesgos que afectarían eventualmente la certidumbre jurídica.

\section{Conclusión}

El tema de la posibilidad de una respuesta correcta única en el razonamiento jurídico puede ser planteado en dos marcos diferentes: uno es el de la inferencia jurídica, entendiendo por tal la deducción de consecuencias a partir de ciertas premisas, utilizando determinadas reglas de derivación; el segundo marco es el de la toma de decisión jurídica, que supone la elección de una alternativa entre varias, con el fin de hacer máximo un valor específico (la certeza jurídica).

El campo jurídico en el que el primer marco tiene especial senti- 
do es el de la interpretación de una norma en el contexto global del sistema. En este entorno la posibilidad de una respuesta correcta única es remota, sino inexistente, ya que el sistema de normas no es un sistema axiomatizado sino natural, y en consecuencia, cada intérprete puede ( intérprete, lo que naturalmente producirá conclusiones diversas. Sin embargo, tales conclusiones pueden ser ambas coherentes respecto de su cuerpo de premisas. En este sentido resulta particularmente útil considerar el concepto de «solución única» en la Teoría de la Acción Racional, ya que dicha solución ha probado ser (mediante las investigaciones de Nash y Kalai-Smorodinski) única sólo respecto de un determinado set de restricciones y reglas. Como puede verse, la posibilidad de una respuesta correcta única en el campo de la inferencia jurídica queda reducida al caso trivial de que todos los intérpretes coincidan de una vez y para siempre en la selección de las premisas, y también en la aceptación de las reglas de derivación. Resulta innecesario argumentar acerca de lo infrecuente de semejante caso.

El entorno en el que cobra especialmente sentido lo que hemos llamado marco de la decisión jurídica, es la producción judicial y legislativa. Aquí la evaluación de la coherencia de la inferencia sólo funciona como un insumo de la toma de decisión, pero no es suficiente para evaluar la justificabilidad de la misma Por esta razón nuevos elementos o bases materiales de la decisión a los que puede llamarse (como Atienza) con el nombre genérico de «pruebas» son incorporados en el escenario de la justificación, lo que como es natural aleja toda posibilidad de respuesta correcta única. Porque si es infrecuente que los intérpretes coincidan en la aceptación de las premisas y la regla, la probabilidad de que ellos también acuerden acerca del valor de los elementos materiales a considerar en una decisión, definitivamente tiende a cero.

El hecho de que no exista una respuesta correcta única en el marco del razonamiento jurídico, no implica renunciar a la posibilidad de justificación del mismo. En el caso de la inferencia jurídica, dicha justificación coincide con el análisis de la coherencia que guardan las consecuencias con las premisas y reglas utilizadas. En el caso de la decisión jurídica, el análisis de la coherencia, siendo necesario no es suficiente. En este entorno particular, a fin de determinar cuál es la justificación aceptable, es necesario en primer lugar acordar acerca del carácter y de la finalidad de la decisión que se elabora. Si acordamos (como parece ser la tendencia generalizada) que la decisión jurídica es una 
decisión racional interactiva bajo incertidumbre, cuya finalidad es la de maximizar la certeza jurídica, tendremos que aceptar que el momento de construcción y de justificación son inseparables, ya que como la instancia de validación se encuentra ubicada en el propio momento de elaboración de la decisión, no existe ningún razonamiento posterior a la decisión misma que pueda justificarla.

En cuanto a los mecanismos de justificación, los mismos deberán tender a hacer más transparentes y ampliamente argumentadas las alternativas que se ofrecen al decisor a fin de que el mismo pueda corregir oportunamente su probabilidad a priori. En este sentido el uso de la interacción (con inclusión del diálogo), y de la publicidad de la misma, son los modos más apropiados; pero sólo si se toma en consideración que el momento en que tales mecanismos son utilizados es una condición crucial para la justificación, ya que la finalidad de los mismos es la de aportar información para la construcción de la decisión y no información acerca de una decisión ya concluida.

\section{BIBLIOGRAFÍA}

* Aarnio, A. (1989), One Right Answer and the Regulative Principle of Legal Reasoning, International Seminar on Legal Theory. Universidad de Alicante.

* Atienza, M. (1989), Outlines of a Theory of Legal Argumentation, International Seminar on Legal Theory. Universidad de Alicante.

Barragán, J. (1989-a), «La Inferencia Jurídica», Revista de la Facultad de Ciencias Jurídicas, núm. 73 , Caracas.

_ (1989-b), La toma de decisiones interactivas, Friedrich Ebert Stifturig.

De Finetti B. (1989), La logica dell'incerto, Roma (de la versión francesa/ 1937).

Harsanyi, J. (1988), Decisión and Game Theoretic Models in Utilitarian Ethics, Center for Research in Management, Berkeley.

Kalai, E., y Smorodinski, M. (1975), «Other Solutions to Nash's Bargaining Problem», Econometrica, vol. 43,3 .

Nash, J. (1950), «The Bargaining Problem», Econometrica, 18.

Press, James (1939), Bayesian Stalistics, John Willey, N. York.

Savage, L. J. et al (1970), The Foundations of Statistic Injerence-A Discussion, Methuen \& Co. London.

* Se trata de los dos trabajos publicados en este mismo número de DOXA. 